\title{
SURGICAL TIPS
}

\section{Fat injections made quick and easy}

\author{
Michael Bell MD FRCSC FACS
}

Cat injections are a well-established and credible technique

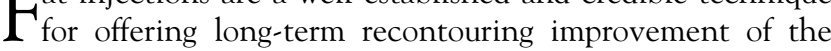
aging face and even for selected depressions from scarring (1).

The techniques for doing this have been described by various authors; many of these techniques require a most extensive attention to detail (2).

The author independently worked out a fat injection technique approximately 15 years ago, which has proven to be quite efficient, simple and effective, with results that he feels are compatible with other reports in the literature.

The efficiency of the technique relates to utilizing a $60 \mathrm{~mL}$ Toomey syringe with a single port $3 \mathrm{~mm}$ Tulip cannula (Tulip Products Inc, USA). This allows harvesting of large volumes of fat quickly from an area that has been anesthetized with tumescent solution, usually the abdomen. The $60 \mathrm{~mL}$ syringe readily allows removal of approximately $200 \mathrm{~mL}$ to $300 \mathrm{~mL}$ of good quality fat as required under low pressure. The pressure is kept

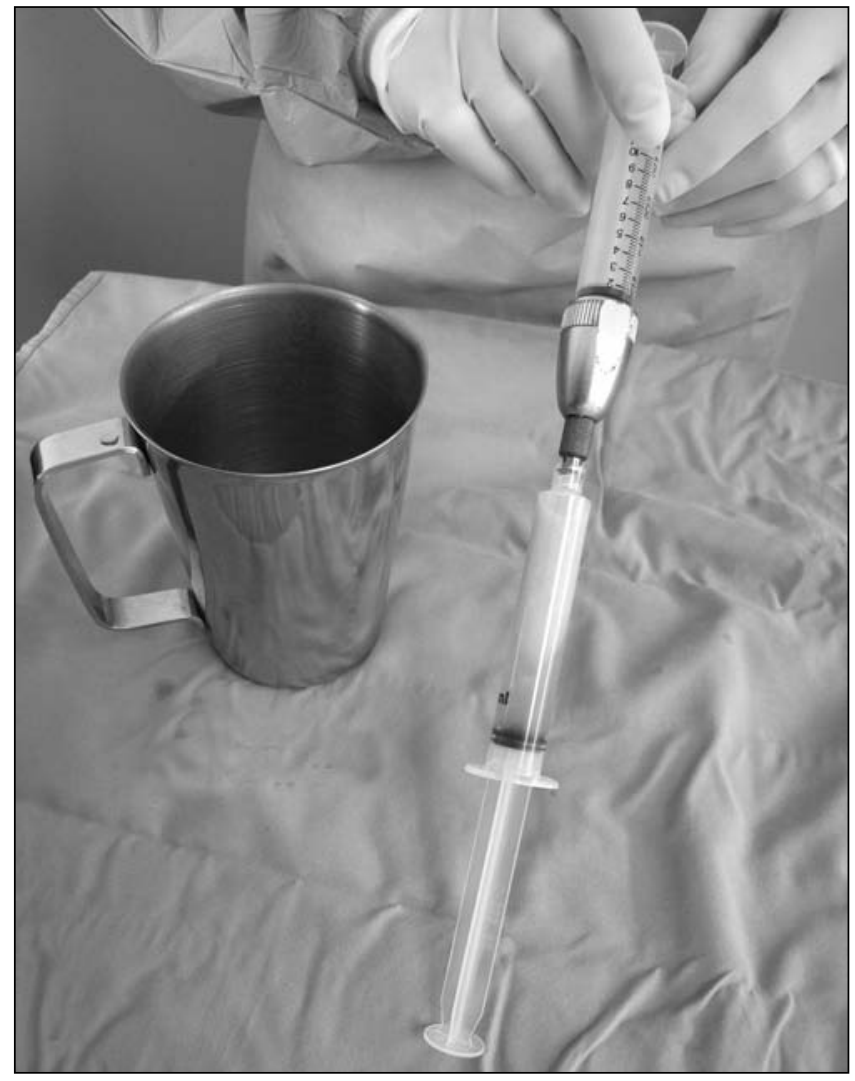

Figure 1) Transferring fat from a medication syringe to a LuerLok syringe. Note the tall container low by setting a block on the syringe handle to remove only 20 $\mathrm{mL}$ to $30 \mathrm{~mL}$ of fat at a time.

The fat is then simply placed in a tall stainless steel container of saline. The fat floats to the top and is separated readily by aspiration into a $10 \mathrm{~mL}$ medication syringe. The medication syringe is used to inject the fat into a LuerLok $10 \mathrm{~mL}$ syringe via a Tulip transfer tube (Tulip Products Inc, USA), which also emulsifies it (Figure 1). The LuerLok syringe is then capped with a 25 gauge needle and placed in an oldfashioned open centrifuge (Figure 2), which is spun for approximately $30 \mathrm{~s}$. This separates the pure fat so that it is then readily injected through a 14 gauge needle or cannula.

The fat is placed in layers on contour depressions to ensure its best take, as detailed by other authors $(3,4)$.

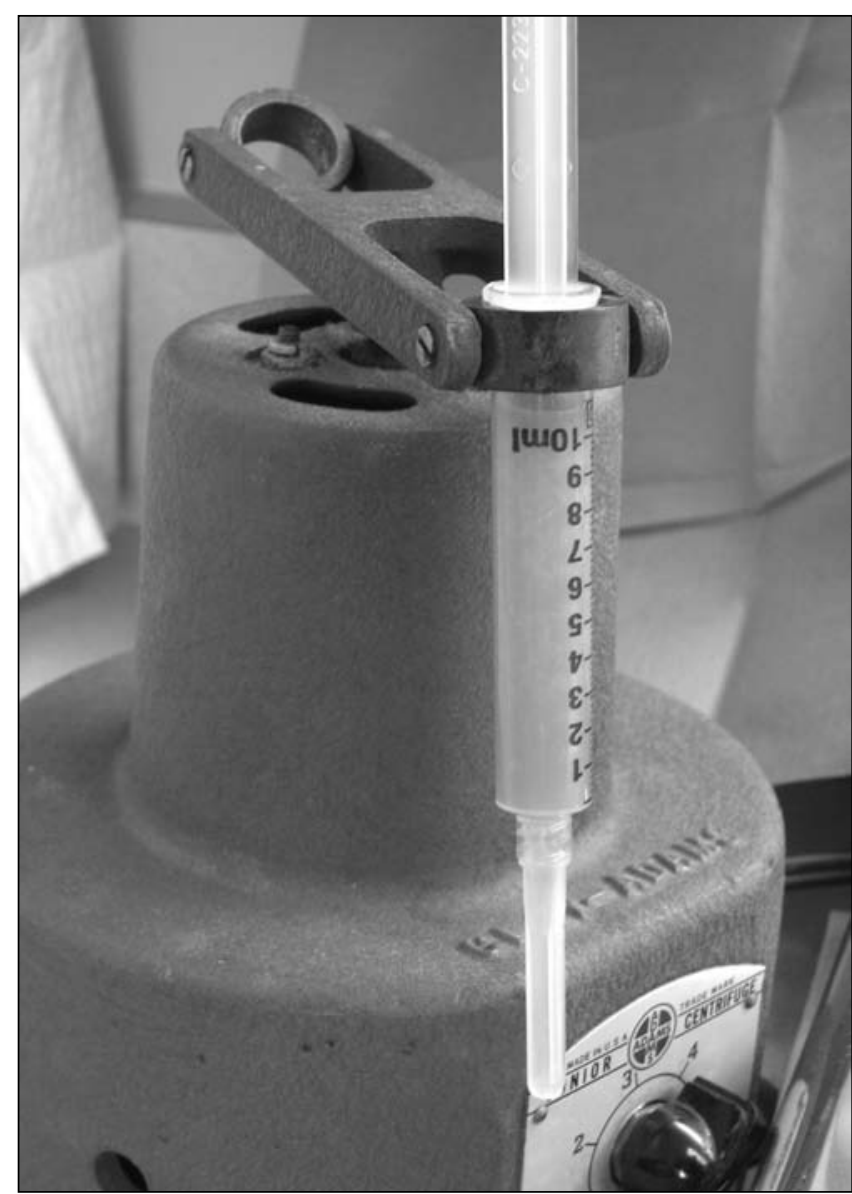

Figure 2) LuerLok syringe to be spun in a centrifuge

The Ottawa Hospital, Ottawa, Ontario

Correspondence and reprints: Dr Michael Bell, The Ottawa Hospital, 402 - 1919 Riverside Drive, Ottawa, Ontario K1H 1 A2.

Telephone 613-739-5424, fax 613-739-7168, e-mail msgbell@cyberus.ca 
For fine tuning fat placement such as along the infraorbital rim and the lateral oral commissures, a 14 gauge steel needle can be used. For safety, the tip of the needle can be blunted somewhat with a file, and then simply bent so that the point is within the lumen of the needle itself. The needle is now essentially a blunt cannula. It can be readily passed through the subcutaneous tissue without worry of tissue damage or bleeding.

Division of the small tethering fibres beneath the nasolabial folds and the lateral oral commissures is simply done with a small handmade instrument. A skin hook can be cut short where the shaft diameter is about $2 \mathrm{~mm}$. If the metal is simply hammered flat in a vise and the flattened end then notched with a file and properly sharpened, an excellent instrument can be fabricated within a few minutes, with very little effort (Figure 3 ).

The only complications the author has encountered from fat injections are three patients who developed somewhat discrete firm lumps on the upper lip and in the area of the orbital rim. These were small (less than $1 \mathrm{~cm}$ in size) and were fairly firm, almost like a lima bean in consistency. The true nature of these has never been determined, but all three were simply removed by using a blunt 14 gauge needle pushing and pulling and rotating the tip while aspirating with a $3 \mathrm{~mL}$ syringe. This worked, in essence, like liposuction removal of soft tissue in other areas, and was successful in each case. Clinically, the lumps appeared to contain fibrofatty tissue but were not histologically examined. There were no hematomas or infections.

The advantage of the above-mentioned technique is that it is more efficient because it harvests a large volume of fat and avoids transferring fat into sterile tubes before centrifuging. All

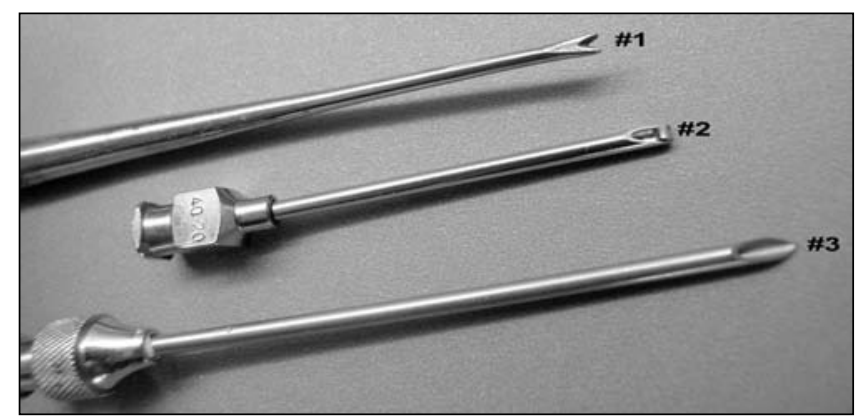

Figure 3) 1 Y dissector; 2 Blunt needle, 14 or 16 gauge; 3 Sharp needle, 14 gauge

injections are done with a $10 \mathrm{~mL}$ syringe and 14 gauge needle blunt or sharp. This relatively macro technique seems to work effectively with far less time and effort, and with very satisfactory results. To date, the author has performed fat injections in over 1200 patients in the past 10 years.

\section{REFERENCES}

1. Guerrerosantos J. Simultaneous rhytidoplasty and lipoinjection: A comprehensive aesthetic surgical strategy. Plast Reconstr Surg 1998;102:191-9.

2. Carraway JH, Mellow CG. Syringe aspiration and fat concentration: A simple technique for autologous fat injection. Ann Plast Surg 1990;24:293-6.

3. Coleman SR. The technique of periorbital lipoinfiltration. Operative Techniques Plast Reconstr Surg 1994;1:120.

4. Loeb R. Nasolabial fold undermining and fat grafting based on histological study. Aesthetic Plast Surg 1991;15:61-6. 